\title{
Pancreatic progenitor cells from the islets of diabetic monkey models
}

\author{
Chunlin Zou ${ }^{1}$, Zeyu Wang ${ }^{2}$, Alex Zhang ${ }^{1}$ \\ ${ }^{1}$ Cell Therapy Center, Beijing Institute of Geriatrics, Xuanwu Hospital, Capital Medical University, China; ${ }^{2}$ Wincon TheraCells \\ Biotechnologies Co., China
}

Despite recent success in islet transplantation for the treatment of type 1 diabetes, the lack of a surrogate source of donor cells that can express insulin remains a major obstacle. The present study aims to identify stem cells/ progenitors directly from adult monkey islets and to assess the proliferation and differentiation potential of such cells. Our results indicate that there are pancreatic progenitor cells in the adult pancreatic islets in both normal and type 1 diabetic animals. The isolated pancreatic progenitor cells can be greatly expanded in culture. Upon the removal of growth medium, these cells spontaneously form islet-like cell clusters (ICCs), which could be further induced to secrete insulin by inductive factors. Furthermore, the secretion of insulin and C-peptide from the ICCs responds to glucose and other stimuli, indicating that the differentiated cells not only resemble $\beta$-cells but also possess the unique biological function of $\beta$-cells. In our initial autologous transplantation experiments in which ICCs were infused into the portal vein of the liver of diabetic monkeys, the elevated blood glucose levels were lowered for extended periods of time, accompanied by an increase of both insulin and $\mathrm{C}$-peptide levels in the blood. This study provides a foundation for further characterization of adult pancreatic progenitor cells and autologous transplantation using pancreatic progenitor cells in treating diabetic monkeys.

Keywords: type 1 diabetes, progenitor cells, autologous transplantation, primates

Cell Research (2008) 18:s173. doi: 10.1038/cr.2008.263; published online 4 August 2008

Correspondence: Alex Zhang

E-mail: yaz@bjsap.org

Alex Zhang, PhD, Dr Zhang received his BA from University of Science and Technology of China and PhD in cell biology from Northwestern University (USA). Following a postdoctoral fellowship at Stanford University, he took the position as director of Cell Therapy Center at Xuanwu Hospital of Capital Medical University in Beijing. His research interest has been on animal development and stem cell biology, and his work has been published in top scientific journals such as Nature, Neuron and Development. His current research focuses on using nonhuman primate models to test stem cell based technology in treating degenerative diseases. 